\title{
Discussion
}

of

"Ten Years Experience with the Swiss National Bank Monetary Policy Strategy" by T. Jordan, M. Peytringnet and E. Rossi

GeORG ICH $^{\mathrm{a}}$

\section{General Comments}

Jordan, Peytrignet and Rossi (JPR) present a very concise and informative description, analysis and assessment of the SNB's new policy strategy adopted at the end of 1999. I my view, their paper is required reading for anyone interested in Swiss monetary policy since the beginning of the twenty-first century. They conclude that the new strategy was successful: The SNB largely achieved its main objective of preserving price stability. Moreover, the new concept proved its worth during the current financial crisis.

I broadly agree with JPR's conclusions. The new strategy has improved the SNB's policy performance as compared with monetary targeting pursued until 1999. A major advantage of the new strategy is that it prompts the SNB to react pre-emptively to shocks - notably cyclical shocks - threatening to jeopardize price stability. Under monetary targeting, the SNB frequently failed to respond adequately to cyclical shocks to price stability because this policy approach did not trigger interest rate movements strong enough to stabilize cyclical movements in the price level. ${ }^{1}$ However, decisive and symmetric response to cyclical shocks is essential if the SNB is to maintain price stability. Since 1999, the SNB, on the whole, has behaved in this fashion.

Sections 9 and 10 of JPR's paper provide a very useful summary and assessment of the measures taken by the SNB after the outbreak of the financial crisis in August 2007. I concur with their view that the SNB has successfully met the various challenges posed by the financial crisis. If I take issue with JPR's analysis at all, it concerns a minor point: Why does the SNB regard interventions on the foreign exchange market as an unconventional policy measure? Admittedly, the mostly unsterilized interventions launched in March 2009 were unprecedented,

a University of Bern, private address: Parkweg 7, CH-5000 Aarau, Switzerland.

1 See Rich (2007) for a detailed analysis of this issue. 
at least in terms of their scale. Furthermore, the SNB had not intervened on the foreign exchange market for fifteen years. In the past, foreign-exchange purchases designed to counter an unwanted appreciation of the Swiss franc had frequently conflicted with the SNB's objective of achieving or maintaining price stability. They led to an excessive increase in the money supply threatening to fuel inflation. The SNB could of course have sterilized the purchases of foreign exchange in an effort to preserve price stability. However, the SNB time and again was compelled to conclude that sterilized interventions were ineffective in curbing an unwanted appreciation of the Swiss franc. ${ }^{2}$ The situation in March 2009 was unusual in that the SNB faced both a prospect of serious deflation and an undesirable appreciation of the domestic currency exacerbating the deflation threat. In these circumstances, the $\mathrm{SNB}$ responded sensibly by conducting unsterilized interventions on a large scale.

\section{Forecasting under the New Strategy}

JPR do not consider an issue that concerned the SNB a great deal before the switch to the new strategy. The SNB was not sure whether its forecasting abilities would be good enough to justify adopting an approach that would assign a central role to inflation forecasts in setting monetary policy. Upon the switch to the new strategy, the SNB strove to beef up its forecasting procedures. Therefore, the question arises whether the SNB's forecasts have become more accurate since 2000 .

Under the new strategy, the SNB produces and publishes three-year-ahead forecasts of inflation. It also announces one-year-ahead forecasts of real GDP. These forecasts are established on a quarterly basis. Under monetary targeting, the SNB only produced one-year-ahead forecasts for inflation and output (see RICH, 2007). Moreover, it established its forecasts solely once a year in December. For this reason, I compare the forecasts produced before 2000 with the oneyear-ahead forecasts published in December 2000 and subsequent years.

The SNB occasionally publishes its forecasts in the form of a range, rather than a single figure. For my analysis, I convert forecasts published as a range into a single figure. A forecast of, say, "1.5-2 percent" or "more than 2 percent" is converted to 1.7 or 2.1 percent respectively.

2 Standard macroeconomic models, both with and without rational expectations, suggest that sterilized interventions are ineffective in influencing the exchange rate if international capital flows are highly mobile, which is certainly the case in Switzerland. 
Figure 1: Actual and Forecasted Values of CPI Inflation

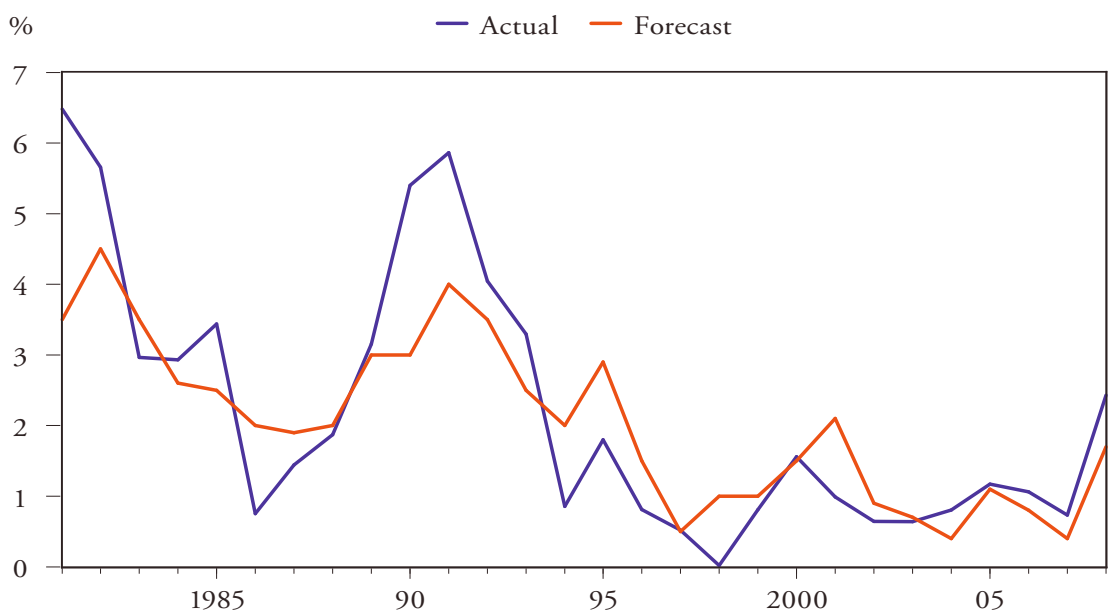

Data sources for the figures and the table: See Appendix

Figure 1 clearly indicates that the SNB's inflation forecasts have become more accurate since 2000. Before the mid-1990s, the SNB tended to underestimate the cyclical amplitude of inflation. Prior to 1991, it also underestimated the inflation trend, as indicated by the positive mean forecast error of $0.56 \%$ shown in Table 1 , which exhibits the forecast errors (actual minus forecasted values) for inflation and real GDP, both in terms of their means and standard deviations (SD).

Figure 2 shows the actual and forecasted values for real GDP. I do not present data for the pre-1991 period because of a break in the available series on real GDP in 1991. Furthermore, up to 1984, the SNB forecasted growth in GNP or final demand, rather than GDP. Contrary to the evidence on inflation, the forecasts for real GDP for the period after 2000 are not better, at least not than those for the period 1991-99. ${ }^{3}$

To sum up, the SNB's forecasts of inflation improved considerably after 1999. In particular, the SNB was more successful in predicting cyclical movements in inflation than before 2000. This may help explaining why the SNB under the new strategy has managed to quell cyclical movements in the inflation rate.

3 However, the available evidence suggests that the forecast errors for the period prior to 1991 were much larger than those for either the periods 1991-1999 or 2000-2008. This is evident if the pre-1991 forecasts are compared with their actual values, as shown in RicH (2007, p. 292). 
Table 1: Forecast Errors for Inflation and Real GDP (\%)

\begin{tabular}{llcc|cc}
\hline \multirow{2}{*}{ Regime } & \multicolumn{2}{c|}{ CPI Inflation } & \multicolumn{2}{c}{ Real GDP } \\
& & Mean & SD & Mean & SD \\
\hline $1981-90$ & Annual monetary target & 0.56 & 1.33 & & \\
$1991-99$ & Medium-term monetary target & -0.10 & 1.02 & -0.27 & 0.97 \\
$2000-08$ & New strategy & 0.05 & 0.52 & 0.41 & 1.20 \\
\hline
\end{tabular}

Figure 2: Actual and Forecasted Values of Real GDP

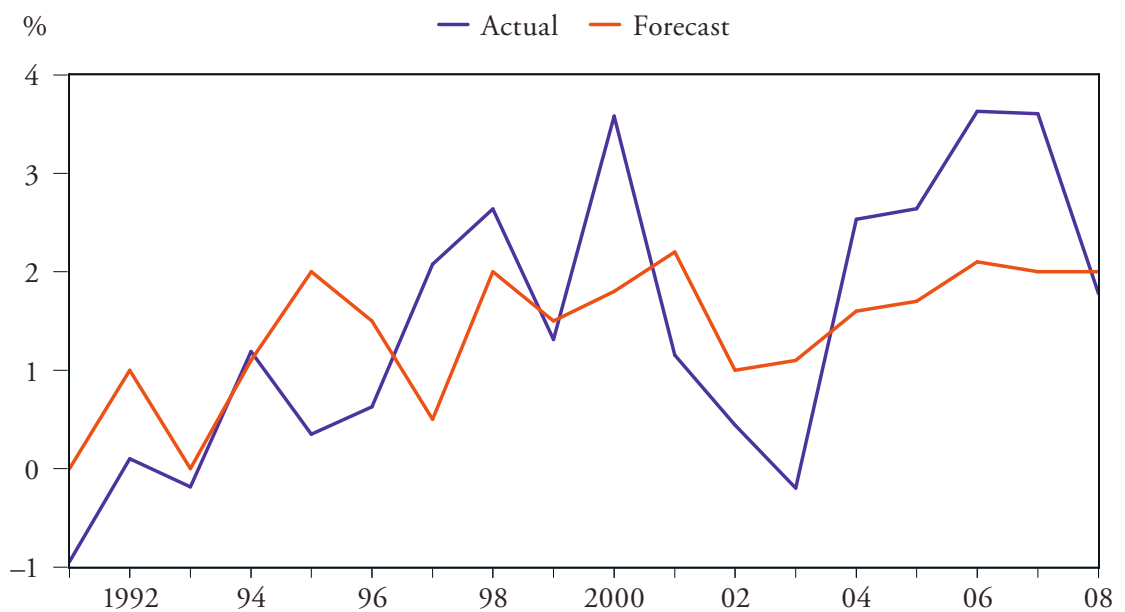

\section{Appendix: Data Sources}

Actual CPI Inflation and real GDP: SNB, Monthly Review.

Forecasts: 1981-1996: RiCH (2007, p. 292).1997-2008: SNB, Quartalsheft, 4/1996, p. 289; 4/1997, p. 258; 4/1998, p.9; 4/1999, p.4; 4/2000, p. 4; 4/2001, pp. 4, 39; 4/2002, p. 9; 4/2003; pp.32, 40; 4/2004, pp. 21, 36; 4/2005, pp. 5, 37; 4/2006, pp. 9, 36; 4/2007, pp. 9, 39. 


\section{Reference}

Rich, Georg (2007), "Swiss Monetary Targeting 1974-1996: The Role of Internal Policy Analysis”, Swiss Journal of Economics and Statistics, 143, pp. 283-329. 